\title{
Improving Antimicrobial Use: Longitudinal Assessment of an Antimicrobial Team Including a Clinical Pharmacist
}

FOREST W. ARNOLD, DO; L. CLIFFORD McDONALD, MD;

DAVID NEWMAN, BS; R. SCOTT SMITH, BS; and JULIO A. RAMIREZ, MD

\begin{abstract}
BACKGROUND: Inappropriate antimicrobial utilization in hospitalized patients has been associated with adverse effects, emergence of resistant bacteria, and increased health care cost. Participation of clinical pharmacists, working as an integral part of a hospital antimicrobial management team (AMT), has been shown to improve antimicrobial use; however, the long-term impact of such a team on antimicrobial use is unclear.
\end{abstract}

OBJECTIVE: Our primary objective was to evaluate whether the number of recommendations to improve antimicrobial use made by a hospital AMT decreased over time. Our secondary objective was to identify and evaluate the acceptance of AMT recommendations with respect to the clinical service, site of infection, and category of suboptimal use.

METHODS: We retrospectively reviewed antimicrobial utilization data collected by the team for the 3-year period from July 1996 to June 1999 at the Veterans Affairs Medical Center in Louisville, Kentucky. The total number of antimicrobial treatment episodes and the number of recommendations were grouped into periods of 6 months each during the 3 years. The type of recommendation, type of infection, and clinical service (medicine versus surgery) were reviewed for the entire 3-year period.

RESULTS: The number of antimicrobial treatment episodes for each of the 6-month consecutive periods was $404,526,406,549,507$, and 612 . The proportion of episodes requiring team recommendations was constant over the 5 consecutive periods: $39 \%, 37 \%, 36 \%, 36 \%, 35 \%$, and $37 \%$. $(P=0.8)$. Acceptance rates of AMT recommendations by the internal medicine and general surgery services remained stable over the length of the study, $84 \%$ and $69 \%$, respectively. The distribution of patients treated by the site of infection also remained stable over the study period.

CONCLUSION: Our results demonstrate that despite the long-term presence of an AMT, the proportion of antimicrobial episodes requiring intervention and the percentage of accepted recommendations remained constant over a 3-year period. Having new resident physicians in teaching hospitals or staff turnover in managed care organizations may necessitate the continued presence of an active AMT.

KEYWORDS: Antibiotic utilization, Antimicrobial management team, Guidelines

J Manag Care Pharm. 2004;10(2):152-58
I nappropriate antimicrobial utilization in hospitalized patients has been associated with adverse effects (e.g., antibiotic-associated diarrhea, acute renal failure), emergence of resistant bacteria, and increased health care cost. ${ }^{1,2}$ The incidence of inappropriate antimicrobial use varies depending on the institution, service, and specific antibiotic and may reach rates as high as $50 \%$ in some institutions. ${ }^{3-8}$

In an effort to decrease inappropriate use of antimicrobial agents, different strategies have been developed, including formulary restriction, use of clinical pathways or guidelines, didactic sessions provided by physicians or pharmacists to health care providers, changing intravenous antimicrobials to an oral form based on clinical and microbiological results (streamlining), computer-assisted management, and multidisciplinary approaches (e.g., staff contributions from infectious diseases, pharmacy, the emergency room, a quality-assurance reviewer, and an administrator), all with various levels of success. ${ }^{9.15}$ Recommendations on how to improve antimicrobial use in hospitalized patients were proposed in 1988 by the Infectious Diseases Society of America (IDSA). ${ }^{16}$

Following these recommendations, we developed a multidisciplinary hospital antimicrobial management team (AMT) in 1989 composed of members from pharmacy, infectious diseases, microbiology, and infection control. The development and implementation of our AMT was presented at the Interscience Conference on Antimicrobial Agents and Chemotherapy workshops during the years 1995 to $1999 .{ }^{17}$ In recent statements by the IDSA and the Society for Healthcare Epidemiology in America, the formation of a performance improvement multidisciplinary team has been recommended as

\section{Authors}

FOREST W. ARNOLD, DO, is a lecturer; L. CLIFFORD McDONALD, MD, was an assistant professor; and JULIO A. RAMIREZ, MD, is a professor, University of Louisville, Department of Medicine, Division of Infectious Diseases, Louisville, Kentucky. (McDonald is now at the Centers for Disease Control and Prevention, Atlanta, Georgia.) DAVID NEWMAN, BS, and R. SCOTT SMITH, BS, are clinical pharmacists, Department of Pharmacy, Veterans Affairs Hospital, Louisville, Kentucky.

CORRESPONDING AUTHOR: Forest W. Arnold, DO, University of Louisville, Dept. of Medicine, Division of Infections Diseases, 512 S. Hancock St., Carmichael Bldg., Rm. 208E, Louisville, KY 40292. Tel: (502) 852-5131; Fax: (502) 852-1147; E-mail: f.arnold@louisville.edu

Copyright $\odot$ 2004, Academy of Managed Care Pharmacy. All rights reserved. 
a tool to minimize inappropriate antimicrobial use and the development of bacterial resistance. ${ }^{18}$

The improvement of antimicrobial utilization by a performance improvement program requires ongoing commitment from hospital administration to dedicate the necessary time and resources to members of the team. Resources to support the full-time commitment of at least 1 clinical pharmacist and the part-time commitment of 1 infectious diseases specialist are necessary for such multidisciplinary teams. Although this involves a financial obligation by the institution, studies indicate that the cost savings associated with antimicrobial streamlining and prevention of bacterial resistance approximate the salary cost of a clinical pharmacist. Combined with the cost of complications due to inappropriate antimicrobial use, the cost savings of an AMT outweigh the expense. ${ }^{19,20}$

Lipsky et al. showed that, following discontinuation of an educational antimicrobial program, there is often a rapid return to the baseline level of inappropriate utilization, indicating that persistent education is necessary for sustained improvement of antimicrobial use. ${ }^{21}$ Nevertheless, in institutions with a wellestablished AMT that performs continuous antimicrobial education and intervention, a decrease in the level of suboptimal antimicrobial use may be expected to occur over time as physicians reach a learning threshold. If the impact of an AMT decreases over time, the cost savings will also likely decrease. At a certain point, the hospital cost of maintaining the program may be perceived to be greater than the savings associated with the program. When this point is reached, hospital administration may choose to shift the resources toward other hospital performance improvement programs. To date, there are no reports in the literature to define whether the proportion of antimicrobial use that is improved by team intervention remains stable over time.

The primary objective of this study was to determine if the number and percentage of antimicrobial interventions by our hospital AMT changed over time. Understanding that the team recommendations would have no impact unless they were accepted, the secondary objective involved evaluation of physician acceptance over the 3-year period. We evaluated the acceptance of team recommendations with respect to the site of infection, clinical service, and category of suboptimal use.

\section{Materials and Methods}

\section{Antimicrobial Team Evaluation}

All hospitalized patients at the Veterans Affairs Medical Center in Louisville, Kentucky, who were prescribed intravenous antimicrobials for the treatment of infections during a 3-year period, were evaluated by members of the hospital AMT. The team evaluated antimicrobial orders written by approximately 60 internal medicine residents, led by 48 attending physicians, and 45 general surgery residents, led by 24 attending physicians. A clinical pharmacist identified the patients receiving antimicrobials and then evaluated the use with an infectious disease physician-specialist, based on policies that had been developed with infection control personnel (infection control nurses, infectious disease specialists and other physicians, employee health nurses, and representatives from microbiology, construction, and administration) and microbiologists. Use of antimicrobials for surgical prophylaxis was the sole exclusion criterion among patients receiving antimicrobials in this study. All other episodes were included even if antimicrobials were discontinued within 24 hours of a team recommendation.

An episode of antimicrobial use was defined as all antimicrobials used to treat a single episode of infection. For example, ceftriaxone and azithromycin prescribed for a patient with community-acquired pneumonia would be considered 1 episode. Initial antimicrobial use was judged "optimal" if the indication was in compliance with hospital antimicrobial guidelines $^{22}$ (Appendix A). Two examples of "optimal" use include (1) an order for 1 gram of intravenous vancomycin every 12 hours for a suspected methicillin resistant Staphylococcus aureus infection in a 66 kilogram patient with normal renal function and no allergies and (2) an order for 2 grams of nafcillin every 4 hours for a patient with a methicillin-sensitive Staphylococcus aureus infection. These guidelines were developed by members of the hospital AMT, reviewed by the hospital staff, and approved by the hospital executive board. Antimicrobial use outside of the guidelines was defined as "suboptimal." Two examples of "suboptimal" use include (1) an inappropriate length of therapy when using vancomycin after susceptibility results reveal Staphylococcus aureus to be methicillin sensitive and (2) an inappropriate dose when using 2 grams of ceftriaxone once daily to treat a nonmeningeal infection.

\section{Antimicrobial Team Recommendations}

Members of the team performed daily rounds on all hospitalized patients who received antimicrobials to treat an infection. An antimicrobial regimen determined to be suboptimal triggered an automatic recommendation by the hospital AMT with the goal to move antimicrobial use into compliance with hospital guidelines. In addition to discussing a recommendation with the physician, it was recorded in the patient's chart. If the physician accepted the recommendation, then the antimicrobial use was classified as optimal and "compliant after recommendation." If physicians disregarded the team recommendation, there were no consequences.

The AMT considered all suboptimal episodes noncompliant. Whether the recommendation was ignored or rejected, the use was classified as suboptimal and noncompliant. The number of antimicrobial episodes evaluated by the team and the percentage of team recommendations were grouped into periods of 6 months each. A total of 6 periods were evaluated during the 3 years of the study. Data were analyzed using the $\chi^{2}$ statistic (Statcalc function of Epi Info 2000, CDC, Atlanta, Georgia) to 


\section{APPENDIX A Excerpts From the Guidelines for the Use of Antimicrobials}

\section{OBJECTIVES}

Antimicrobial agents are the most important intervention in the therapy of infectious diseases. Their proper use is not only essential for patients to recover from the infectious process, but also to avoid potential toxic effects, emergence of resistant bacteria, and to reduce health care costs.

Guidelines for the use of antimicrobial agents have been developed by the Infectious Diseases Section of the Department of Medicine and the Surgery Department of the University of Louisville School of Medicine in conjunction with the Pharmacy Service at the Louisville Department of Veterans Affairs Medical Center. The goal of these guidelines is to promote optimal antimicrobial use in hospitalized patients. These guidelines are based on national standards and more importantly on local experience. These guidelines after review by the medical center medical staff, have been approved by the Pharmacy and Therapeutics Committee and the Clinical Executive Board to become the policy for the use of antimicrobials in this medical center.

The core of the guidelines is based on

1. Formulary management

2. Pharmacodynamic principles

3. Principles for empiric therapy

4. Principles of known pathogen therapy

5. Principles for switch therapy

6. Principles for prevention and control of nosocomial infections

At this institution, the formulary has evolved stepwise from an open formulary, to a restricted formulary and now for about 7 years, a guidelines-based formulary. This type of formulary is more patient oriented and outcome based. It also has the advantage of both reducing the risk of emergence of resistant pathogens, as well as controlling health care cost. (P\&T. 1996;21:270-77.)

Knowledge of the pharmacodynamics of antimicrobials can be applied to better design regimens to maximize efficacy, decrease toxicity, decrease risk of emergence of resistant bacteria, and decrease cost. Antibiotics that inhibit cell wall synthesis like betalactams and vancomycin have time dependent bactericidal activity, i.,e., as long as the concentration is a certain level above the MIC, the rate of killing is not increased with higher doses. Except for carbapenems, these antibiotics also do not have significant post antibiotic effect (PAE). By contrast, antibiotics that inhibit DNA or protein synthesis such as aminoglycosides and quinolones have concentration dependent bactericidal activity The higher the concentration, the higher the rate of bacterial kill. These antibiotics also possess a prolonged PAE (Infect Control Hosp Epidemiol. 1990;11:319.)

Infection control plays a major role in the surveillance of nosocomial infections and in the prevention of their spread. Also important, is its role in employee health to prevent infection from patients or the hospital environment.

\section{CLASSIFICATIONS OF THERAPY}

Clinical situations in which antimicrobial agents are regularly used are defined as follows:

1. Prophylactic Use

2. Therapeutic Use

- Empiric Therapy

- Known Pathogen Therapy

- Switch Therapy

Prophylactic Use:

In the clinical situation of "prophylactic use," the antimicrobial is used to prevent infection. In the hospital, the most commonly encountered prophylaxis is in surgery to prevent wound infection. There are also other indications for prophylactic use of antimicrobials, e.g., prevention of bacterial endocarditis, influenza A. Post exposure prophylaxis against HIV infection (needle stick), or meningococcal infections are also outlined in Appendix B.

\section{Empiric Therapy:}

In the clinical situation of "empiric use." the antimicrobial is used as initial therapy directed to eradicate the most likely pathogens. Before initiation of antimicrobials, appropriate specimens for stains and cultures of microorganisms should be obtained. Results of iden tification and susceptibility of microorganisms are likely to be available in the following 48 to 72 hours. The use of broad spectrum antibiotics or combination therapy is usually necessary to cover the different organisms capable of causing an infection. The use of agents in this situation should not extend beyond the time required to obtain results of cultures and susceptibility. Criteria for the use of particular intravenous antimicrobials agents in the clinical situation of "empiric use" are described in Appendix A. Recommendations of empiric therapy for some common infections are outlined in Appendix C.

\section{Known Pathogen Therapy:}

In the clinical situation of "known pathogen use," the antimicrobial is used when the microbiology laboratory has identified the microorganism causing the infection and the susceptibility pattern is known. If during "empiric use," the patient was started on combination therapy or broad spectrum antibiotic, the antimicrobial spectrum should now be narrowed to cover the microorganism identified as the etiologic agent. Antimicrobial ther- apy should be continued with an appropriate narrow spectrum antibiotic to avoid colonization with resistant organisms and superinfection. Criteria for the use of particular intravenous antimicrobial agents in the clinical situation of "known pathogen use" are described in Appendix A.

Switch Therapy-Conversion From IV to PO

In the clinical situation of "switch therapy use", oral antimicrobials replace intravenous usage for completion of therapy. Intravenous therapy is almost always employed in serious infections to ensure maximal serum levels. In patients with infections localized in areas of poor antibiotic penetration (i.e., meningitis, endocarditis), and in patients with immunodeficiency states (i.e, neutropenia), intravenous antibiotics are recommended for the complete duration of therapy. The great majority of patients with infection do not require completion of the antimicrobial course with intravenous therapy. Transition to oral therapy can usually be employed.

The following criteria were developed for transition from parenteral to oral antimicrobial:

a) no clinical indication for intravenous therapy (i.e., meningitis, endocarditis, neutropenia)

b) no clinical indication of abnormal gastrointestinal absorption of drugs (i.e., diarrhea)

c) patient is afebrile for at least 8 hours

d) signs and symptoms related to infection are improving

e) the white blood cell count is normalizing

If the patient meets the above five criteria, the completion of the treatment may be achieved with an oral agent

Oral antimicrobials recommended as alternatives to parenteral therapy are presented in Section IV, The choice of oral agents is guided by pharmacokinetic and pharmacodynamic principles.

III. AVAILABLE ANTIMICROBIAL AGENTS (list available from authors)

IV. ORAL ALTERNATIVES TO IV ANTIMICROBIALS

When clinically indicated, switch from the following parenteral to the oral agent is suggested. (List available from authors; e.g., oral dicloxacillin $500 \mathrm{mg}$ qid for parenteral nafcillin 1 gm q 4 hours.)

V. GUIDELINES FOR THE USE OF COMMON PARENTERAL ANTIMICROBIALS The following guidelines are organized schematically for each main intravenous antimicrobial, with its more common indications. The recommended doses are based on normal renal function. (List available from authors; includes the following example: 6. Cefazolin: To be used in skin and soft tissue infections, and other infections caused by susceptible Staphylococcus aureus. It may be used in urinary tract infections when susceptible gram negative organisms are identified. The high dose regimen should be used for endocarditis. Cefazolin does not penetrate into the blood brain barrier and should not be used to treat meningitis. Recommended Dose: Usual dose: $1 \mathrm{~g}$ q 8h; High dose: 2 g q 8h)

\section{GUIDELINES FOR PROPHYLACTIC THERAPY}

(Complete list available from the authors; includes specific guidelines for use of antimicrobials in surgical procedures, bacterial endocarditis, rheumatic fever, meningococcal disease, tuberculosis prevention)

\section{GUIDELINES FOR EMPIRIC THERAPY FOR SOME COMMON INFECTIONS}

(Complete list available from authors; includes the following for diabetic foot infections, as an example.)

6.2 Diabetic foot infections: Infected diabetic foot ulcers and infected pressure ulcers are most always polymicrobial. Deep tissue cultures provide the most reliable bacteriologic information. The most common pathogens are S.aureus, enterococci, group B streptococci, enterobacteriaceae and anaerobes. Debridement often required.

Oral Therapy:

a. AMOX/CLAVUL $875 \mathrm{mg}$ po bid

b. CIPROFLOXACIN $750 \mathrm{mg}$ po bid alone or in combination with CLINDAMYCIN $300 \mathrm{mg}$ po qid

c. OFLOXACIN $400 \mathrm{mg}$ po bid alone or in combination with CLINDAMYCIN $300 \mathrm{mg}$ po qid

Parenteral therapy:

a. TICAR/CLAVUL $3.1 \mathrm{~g} \mathrm{q} 6 \mathrm{~h}$

b. AMP/SULBACT $3.0 \mathrm{~g} \mathrm{q} 6 \mathrm{~h}$

c. PIP/TAZOBACT $3.375 \mathrm{~g} \mathrm{q} 6 \mathrm{~h}$

Treatment failure is mostly because of poor vascularization of infected and necrotic tissue which would need to be debrided. Rarely, it may be due to resistant pathogens.

An updated version of the guidelines is available at: www.staarprogram.com/Veteransguide.pdf. 
determine significant differences in the proportions of inappropriate antimicrobial use between different time periods.

All episodes defined as suboptimal were divided into 6 categories: (1) inappropriate length of therapy (e.g., length of therapy is either too long or too short according to guidelines), (2) inappropriate dose (e.g., dose too low or too high relative to the patient's renal function and dose recommended by guidelines), (3) more effective drug available (e.g., using vancomycin for therapy of methicillin susceptible Staphylococcus aureus), (4) less toxic drug available, (5) narrower spectrum drug available, and (6) use unjustified (e.g., using antimicrobials when no infection was identified). All episodes were grouped by clinical service-internal medicine physicians versus general surgeonsand by site of infection-lower respiratory infection, urinary tract infection, skin and soft tissue infection, intra-abdominal infection, and "other."

\section{Results}

A total of 3,004 antimicrobial treatment episodes were evaluated by the AMT during the 3-year study period, of which 1,103 (36.7\%) were considered suboptimal and received recommendations. Of those recommendations, 890 (81\%) were accepted. There was no significant variation in this proportion of treatment episodes requiring AMT recommendations between the 6 -month periods over the 3 years $(P=0.8$, Figure 1$)$ In contrast to the proportion of treatment episodes requiring recommendations, there was variation in the proportion of recommendations that were accepted, with significantly more accepted during the two 6-month periods in 1997 than in the three 6-month periods of 1998 to $1999(P<0.0001)$.

Acceptance of a recommendation for a more effective or less toxic drug was more likely than acceptance of any other category of recommendation $(P<0.0001$, Table 1$)$. By type of service, the proportion of antimicrobial treatment episodes that were suboptimal did not differ between medicine $(37 \%)$ and surgery (35\%), $(P=0.57$, Table 2$)$, but general surgery services were less likely (71\%) than internal medicine services (83\%) to accept antimicrobial recommendations $(P=0.009)$.

There was no significant variation in the proportion of recommendations accepted by site of infection $(P=0.15$, Table 3$)$. Among all antimicrobial episodes, 29.6\% (890 of 3,004) were improved after intervention by the AMT. The acceptance rates of AMT recommendations by internal medicine or general surgery service remained stable over the study period, at $83 \%$ and $71 \%$, respectively (Table 2). The distribution of the infections treated during each study period remained stable, with lower respiratory tract infections as the predominant infection over the 3-year period (Table 3).

\section{Discussion}

With understanding and support from hospital administration, a well-established and recognized AMT has been maintained in our hospital over the past 10 years. One might expect that an active AMT would, over time, have the effect of reducing the proportion of infection episodes associated with suboptimal use of antimicrobials. Our study indicates that in a teaching hospital, the proportional improvement in antimicrobial use achieved by implementation of a hospital AMT remained stable over time. Over a 3-year period, we were unable to document any decrease in the proportion of antimicrobial episodes that were suboptimal prior to team recommendations.

One possible explanation for this finding is the presence of both medical and surgical resident training programs in our institution. The involvement of new resident physicians every year in the care of hospitalized patients may require the continued educational presence of an AMT. In addition, our study revealed a continued benefit from the active interventions made by the AMT. Active intervention by a team of clinical pharmacists and a physician-specialist in infectious disease over a 3-year period achieved the following outcomes: (a) 35\% of all antimicrobial use was identified as suboptimal compared with clinical guidelines and (b) $81 \%$ of recommendations to improve suboptimal use were accepted.

The success of a hospital program based on team recommendations depends greatly on medical staff acceptance of a team member's advice, as the mere recognition of inappropriate use without successful intervention will not improve patient outcomes. In our hospital, recommendations are always based on institutional antimicrobial guidelines, always documented, and, in some cases, performed face-to-face with the primary physician. In our institution, an average of $81 \%$ of all recommendations to optimize antimicrobial use were accepted. Our strategy of using real-time utilization review and continuous education may explain the high level of acceptance documented over time in our institution. The well-established nature of the team, including contributions by the clinical pharmacist working in close collaboration with infectious disease physicians, is also a factor in the high level and sustained acceptance rate for AMT recommendations.

Among the categories of suboptimal antimicrobial use, inappropriate dosing was most common and, hence, was the subject of $41 \%$ of all recommendations. By preventing the overdose of antimicrobials, the AMT may improve patient safety by avoiding drug toxicity and side effects. Alternatively, by preventing underdosing, the team may slow the emergence of resistance and speed the recovery of patients or even prevent treatment failure. Together, these factors may quell the need for the development of new agents. Similarly, other team interventions may have contributed to less expensive and more streamlined antimicrobial use. ${ }^{1,2,23}$ According to a recent survey of 14 U.S. hospitals, those with active antibiotic management programs used significantly more of less-expensive groups of antimicrobials compared with those with only passive control policies. ${ }^{24}$ In our hospital, 5 of the 6 categories evaluated by the AMT 


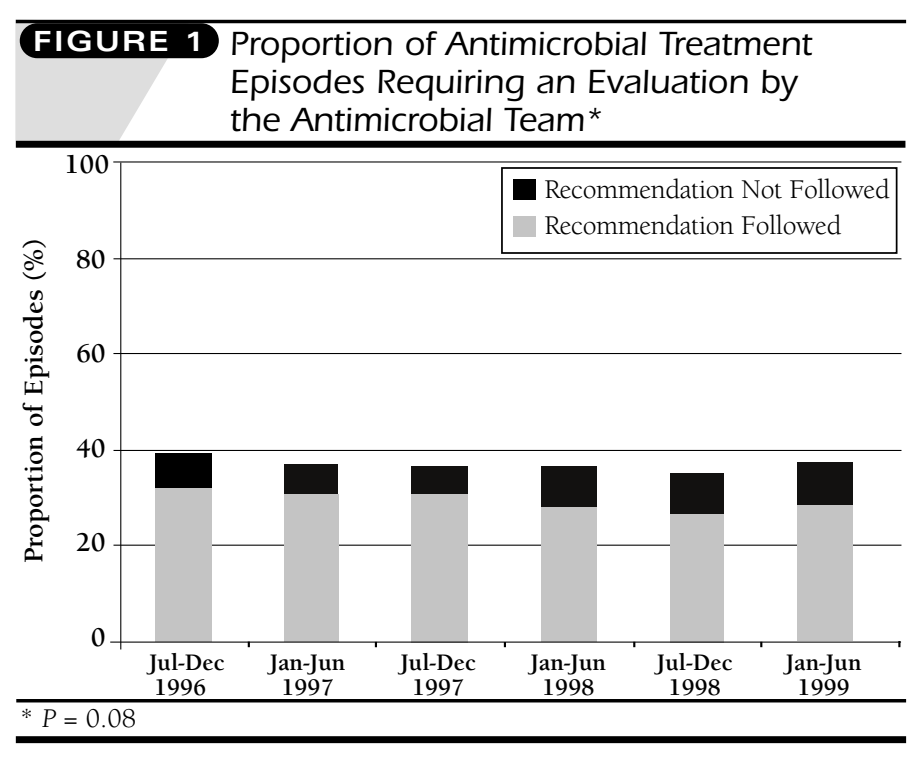

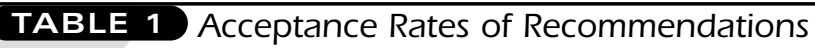
by Category of Suboptimal Use

\begin{tabular}{lcc} 
Category of Suboptimal Use & $\begin{array}{c}\text { No. of } \\
\text { Antimicrobial } \\
\text { Episodes } \dagger\end{array}$ & $\begin{array}{c}\text { No. of } \\
\text { Recommendations } \\
\text { Accepted (\%) } \ddagger\end{array}$ \\
\hline Inappropriate length of therapy & 109 & $78(72)$ \\
Dose inappropriate & 454 & $362(80)$ \\
More-effective drug & 193 & $180(93)$ \\
Less-toxic drug & 36 & $33(92)$ \\
Narrow-spectrum drug & 232 & $181(78)$ \\
Use unjustified & 79 & $56(71)$ \\
\hline Total & 1,103 & $890(81)$ \\
\hline * For the 3-year period July 1, 1996, through June 30, 1999. & \\
$\dagger$ Percentage calculated from suboptimal antimicrobial use; P>0.0001.
\end{tabular}

TABLE 2 Optimal and Suboptimal Antimicrobials Use and Acceptance Rate of Recommendations by Type of Clinical Service

\begin{tabular}{|c|c|c|c|}
\hline Service* & $\begin{array}{c}\text { No. of } \\
\text { Antimicrobial } \\
\text { Episodes } \dagger\end{array}$ & $\begin{array}{c}\text { No. of } \\
\text { Suboptimal } \\
\text { Uses }(\%) \neq\end{array}$ & $\begin{array}{c}\text { No. of } \\
\text { Recommendations } \\
\text { Accepted (\%)§ }\end{array}$ \\
\hline Medicine & 2,277 & $843(37)$ & $703(83)$ \\
\hline Surgery & 727 & $260(35)$ & $187(71)$ \\
\hline Total & 3,004 & $1,103(36)$ & $890(80)$ \\
\hline \multicolumn{4}{|c|}{$\begin{array}{l}\text { * "Medicine" represents internal medicine services; "Surgery" represents general } \\
\text { surgery services. } \\
\dagger \text { For the 3-year period July 1, 1996, through June 30, } 1999 \text {. } \\
\ddagger P=0.57 \text {. } \\
\S \text { Percentage calculated from suboptimal antimicrobial use; } P=0.009 \text {. }\end{array}$} \\
\hline
\end{tabular}

(Table 3) focus on strategies typically associated with using fewer antimicrobials or stopping unnecessary antimicrobials.

One practice that may favor the development of antimicrobial resistance is the repetitive use of a single antimicrobial agent as empiric therapy for most infections. ${ }^{25}$ Hospitals with restrictive antimicrobial formularies that allow the physician to use only a small number of antibiotics for empiric therapy may favor the development of resistant bacteria by such selective antimicrobial pressure. In an attempt to prevent this, we changed from a restrictive drug formulary to a more flexible formulary based on guidelines for optimal antimicrobial use. This type of formulary policy may contribute to a more collegial, less restrictive clinical practice environment and facilitate acceptance of team recommendations. Our hospital antimicrobial guidelines are distributed to all faculty and residents and are the foundation for the team recommendations.

Our data suggest that both surgical and medical services have very similar rates of suboptimal antimicrobial use $(P=0.57$, Table 2$)$. However, general surgical teams appear somewhat less likely to accept team recommendations compared with internal medicine teams, measured by a higher rate of acceptance in medical units $(P=0.009$, Table 2$)$. One possible explanation for this finding is the less frequent face-to-face interaction between our team members and surgeons and residents of the surgical services. The surgeons were relatively less accessible because they performed rounds earlier in the morning than the AMT, and they were often in the operating room during the day.

The acceptance rates of team recommendations did not differ by the site of infection. Among types of suboptimal antimicrobial use, recommendations to discontinue antimicrobials in patients without documented infection had the lowest acceptance rate, while recommendations to give a more effective antimicrobial agent had the highest acceptance rate.

\section{Limitations}

Several limitations of this study should be recognized. Aside from its retrospective design, an important limitation was the need to classify antimicrobial use dichotomously as either "optimal" or "suboptimal." This method may have oversimplified a complex case, especially when so many variables came into play when choosing antimicrobials for a patient, such as allergies, previous antimicrobial history, and course of illness. These extra variables served as confounders. If any of these confounders affected the more popular categories of "duration of use" or "use justified," then the oversimplification would have been magnified.

We did not have a control group, and it is possible that temporal influences might have affected physician behaviors that were not due to the influences of the AMT. For instance, we were not able to control for campaigns by national organizations (e.g., Centers for Disease Control and Prevention) to improve antimicrobial use. Also, since the AMT did not communicate face-to-face with surgeons as often as desired, the AMT may have influenced the internal medicine physicians more than the surgeons. In this study, there were no consequences for the physicians if they continued using antimicro- 


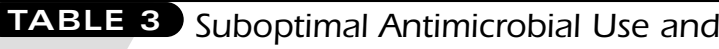 Acceptance of Recommendations by Site of Infection}

\begin{tabular}{|c|c|c|c|}
\hline $\begin{array}{l}\text { Site of } \\
\text { Infection }\end{array}$ & $\begin{array}{c}\text { No. of } \\
\text { Antimicrobial } \\
\text { Episodes* }\end{array}$ & $\begin{array}{c}\text { No. of } \\
\text { Suboptimal } \\
\text { Uses }(\%) \dagger\end{array}$ & $\begin{array}{c}\text { No. of } \\
\text { Recommendations } \\
\text { Accepted }(\%) \ddagger\end{array}$ \\
\hline LRTI & 1,498 & $565(38)$ & 459 (81) \\
\hline UTI & 539 & $226(42)$ & $186(82)$ \\
\hline SSI & 447 & $131(29)$ & $98(75)$ \\
\hline IAI & 222 & $81(37)$ & $61(75)$ \\
\hline Others & 298 & $100(34)$ & $86(86)$ \\
\hline$\overline{\text { Total }}$ & 3,004 & $1,103(37)$ & $890(81)$ \\
\hline \multicolumn{4}{|c|}{$\begin{array}{l}\text { *For the 3-year period July 1, 1996, through June 30, } 1999 . \\
\dagger P=0.0009 . \\
¥ P \text { Percentage calculated from suboptimal antimicrobial use; } P=0.15 . \\
\text { LRTI = lower respiratory tract infection; UTI = urinary tract infection; SSI = skin } \\
\text { and soft tissue infection; IAI = intra-abdominal infection. }\end{array}$} \\
\hline
\end{tabular}

bials inappropriately. Making prescribers accountable for deviation from guidelines may have improved use or at least the acceptance of AMT recommendations over the 3-year period. Finally, the antimicrobial use guidelines underwent independent review performed by the hospital staff and executive committee; the case evaluations performed by the AMT members were not subjected to independent third-party review or other validation.

An important area for future research is the development of interventions that may help to improve the proportion of AMT recommendations that are accepted by physcian-prescribers. It has been suggested that real time, concurrent, or immediate retrospective feedback of antimicrobial use may be an important intervention to improve physician compliance with recommendations. ${ }^{26}$ This study may have been improved if periodic feedback was provided to physician-prescribers, especially if this feedback was shared with department chiefs (peer review) and hospital administration. It would also be valuable in future study designs to measure clinical outcomes (e.g., length of stay, the presence of side effects, utilization of resources, and mortality) for the group of patients with accepted AMT recommendations versus those patients for which AMT recommendations were not accepted. To prove that our data can be generalized, it would be important to develop a national network of hospitals with AMTs to aggregate data on interventions and outcomes.

\section{Conclusion}

Our study supports the need for AMT interventions, over time, at a teaching institution, regardless of the site of infection or the clinical service (medicine or surgery). Over a 3-year study period, the rate of suboptimal use remained fairly constant, at about $35 \%$ of all antimicrobial use. A major implication is that there may be a need for the long-term presence of AMTs, particularly in teaching institutions, where there is regular turnover of medical and surgical residents.

\section{ACKNOWLEDGMENT}

The authors wish to thank Gilbert W. Ritter, RPh, director of pharmacy, Department of Pharmacy, Veterans Affairs Hospital, Lousiville, Kentucky, for reviewing the manuscript.

\section{DISCLOSURES}

No outside funding supported this study. These data were presented, in part, as poster 599 at the 40th annual meeting of the Infectious Diseases Society of America, October 2002, Chicago, Illinois. Author Forest W. Arnold served as principal author of the study. Study concept and design were contributed by authors Julio A. Ramirez, L. Clifford McDonald, R. Scott Smith, and David Newman. Analysis and interpretation of data were contributed by Ramirez, McDonald, Smith, and Newman. Drafting of the manuscript was primarily the work of Arnold and McDonald, and its critical revision was the work of Ramirez. Statistical expertise was contributed by McDonald.

\section{REFERENCES}

1. Glowacki RC, Schwartz DN, Gail SI, Wisniewski MF, Kieszkowski P, Weinstein RA. Antibiotic combinations with redundant antimicrobial spectra: clinical epidemiology and pilot intervention of computer-assisted surveillance. Clin Infect Dis. 2003;37:59-64

2. Sterling TR, Ho EJ, Brehm MS, Kirkpatrick MB. Diagnosis and treatment of ventilator-associated pneumonia -impact on survival. Chest. 1996;110:1025-34.

3. Gilberg K, Laouri M, Wade S, Isonka S. Analysis of medication use patterns: apparent overuse of antibiotics and underuse of prescription drugs for asthma, depression and CHF. J Manag Care Pharm. 2003;9(3):232-37.

4. Gould I, Jappy B. Trends in hospital antibiotic prescribing after introduction of an antibiotic policy. J Antimicrob Chemother. 1996;38:895-904.

5. Anglim A, Klym B, Byers K, Scheld W, Farr B. Effect of a vancomycin restriction policy on ordering practices during an outbreak of vancomycinresistant Enterococcus faecium. Arch Intern Med. 1997;157:1132-36.

6. Thomas M, Govil S, Moses B, Joseph A. Monitoring of antibiotic use in a primary and tertiary care hospital. J Clin Epidemiol. 1996;49:251-54.

7. Thuong M, Shortgen F, Zazempa V, Girou E, Soussy C, Brun-Buisson C. Appropriate use of restricted antimicrobial agents in hospitals: the importance of empirical therapy and assisted reevaluation. J Antimicrob Chemother. 2000;46:501-08

8. Gyssens I, Blok W, van dBP, Hekster Y, van dMJ. Implementation of an educational program and an antibiotic order form to optimize quality of antimicrobial drug use in a department of internal medicine. Eur J Clin Microbiol Infect Dis. 1997;16:904-12.

9. Ramirez J, Srinath L, Ahkee S, Huang A, Raff M. Early switch from intravenous to oral cephalosporins in the treatment of hospitalized patients with community-acquired pneumonia. Arch Intern Med. 1995;155:1273-76.

10. Bui K, Quintiliani R. Antimicrobial switch therapy. Conn Med. 1998; 62:665-68

11. Evans R, Pestotnik S, Classen D, et al. A computer-assisted management program for antibiotics and other anti-infective agents. N Engl J Med. 1998; 338:232-38

12. Schentag J, Ballow C, Fritz A, et al. Changes in antimicrobial agent usage resulting from interactions among clinical pharmacy, the infectious disease division, and the microbiology laboratory. Diagn Microbiol Infect Dis. 1993; 16:255-64

13. Briceland L, Nightingale C, Quintiliani R, Cooper B. Multidisciplinary cost-containment program promoting less frequent administration of injectable mezlocillin. Am J Hosp Pharm. 1988;45:1082-85. 
14. Gross R, Morgan A, Kinky D, Weiner M, Gibson G, Fishman N. Impact of a hospital-based antimicrobial management program on clinical and economic outcomes. Clin Infect Dis. 2001;33:289-95.

15. John J, Fishman N. Programmatic role of the infectious diseases physician in controlling antimicrobial costs in the hospital. Clin Infect Dis. 1997;24:471-85.

16. Marr J, Moffet H, Kunin C. Guidelines for improving the use of antimicrobial agents in hospitals: a statement by the Infectious Diseases Society of America. J Infect Dis. 1988;157:869-76.

17. Ramirez J, Carrico R, Snyder J, et al. A program for improving the use of antimicrobials and preventing bacterial resistance in hospitals. Workshop presented at: 37th Interscience Conference on Antimicrobial Agents and Chemotherapy; September 1999; San Francisco, CA. [Also presented at 33rd to 36th ICAACs].

18. Shlaes D, Gerding D, John JJ, et al. Society for Healthcare Epidemiology of America and Infectious Diseases Society of America Joint Committee on the Prevention of Antimicrobial Resistance: guidelines for the prevention of antimicrobial resistance in hospitals. Clin Infect Dis. 1997;25:584-99.

19. Hunter K, Dormaier G. Pharmacist-managed intravenous to oral stepdown program [discussion 516]. Clin Ther. 1995;17:534-40;

20. Ramirez J. Antibiotic streamlining: development and justification of an antibiotic streamlining program. Pharm Pract Manag Q. 1996;16:19-34.
21. Lipsky B, Baker C, McDonald L, Suzuki N. Improving the appropriateness of vancomycin use by sequential interventions. Am J Infect Control. 1999; 27:84-91.

22. Louisville VA Hospital antibiotic guidelines. Available at: www.staarprogram.com/Veteransguide.pdf. Accessed November 2001

23. Kollef MH. Appropriate empirical antibacterial therapy for nosocomial infections: getting it right the first time. Drugs. 2003;63:2157-68

24. Carling P, Fung T, Coldiron J. Parenteral antibiotic use in acute-care hospitals: a standardized analysis of fourteen institutions. Clin Infect Dis. 1999; 29:1189-96

25. Shlaes DM, Gerding DN, John JF Jr, et al. Society for Healthcare Epidemiology of America and Infectious Diseases Society of America Joint Committee on the Prevention of Antimicrobial Resistance: guidelines for the prevention of antimicrobial resistance in hospitals. Clin Infect Dis. 1997; 25:584-99.

26. Tiley SM, MacDonald JJ, Doherty PL, et al. Active promotion of antibiotic guidelines: an intensive program. Commun Dis Intell. 2003;27(suppl):S13-S18. 